SHORT REPORT

\title{
Transient short free running circadian rhythm in a case of aneurysm near the suprachiasmatic nuclei
}

\author{
K E Bloch, T Brack, A Wirz-Justice
}

\begin{abstract}
A free running circadian rest-activity cycle is rare in sighted individuals living in a normal environment. Even more rare is a periodicity shorter than 24 hours, as observed in actigraphic recordings in a female patient during convalescence after a whiplash injury in a car accident. The documented free running period was 22.5 hours for 19 days. During the subsequent weeks re-entrainment occurred following re-establishment by a social zeitgeber, with a slightly early circadian phase of nocturnal melatonin onset relative to a late sleep period. Magnetic resonance imaging and cerebral angiography showed an aneurysm at the bifurcation of the right internal carotid artery, close to the circadian pacemaker structure (the suprachiasmatic nuclei), which was later occluded.
\end{abstract}

A dvanced sleep phase syndrome is an extremely rare circadian rhythm variant. ${ }^{12}$ It may occur as a familial form related to a missense mutation in a clock component on chromosome 2. ${ }^{3}$ Under conditions without time cues, the circadian rest-activity cycle free runs with a shorter than 24 hour circadian period. Whereas cases of posttraumatic delayed sleep phase syndrome have been observed, ${ }^{4}$ a similar association of central nervous system trauma with advanced sleep phase syndrome has not been reported. We obtained actigraphic recordings of a short period free running circadian rhythm occurring in a female patient after a whiplash injury, which improved after forced resynchronisation by social stimuli and daytime light exposure.

\section{CASE REPORT}

A 43 year old woman was referred for evaluation of excessive daytime sleepiness, disturbed sleep, difficulties in concentration, and intermittent headaches. Twelve months earlier she had suffered from a whiplash injury to the cervical spine in a car accident. Within two weeks after the accident, she noticed excessive sleepiness with an irresistible need to sleep for several hours during the daytime, in addition to eight to 10 hours of night sleep. These symptoms persisted until the time of referral and she was not able to resume her professional activity. She was not following any regular activity, stayed mostly at home, and avoided social contacts. She did not report cataplexy nor hypnagogic hallucinations, and there were no symptoms of mood disorder. She was on no medication and denied taking drugs or excessive amounts of alcohol. Before the accident she had not experienced sleep disturbances, excessive daytime sleepiness, or any other health problems. Her usual sleep period had been between 10 to $11 \mathrm{pm}$ and $6 \mathrm{am}$, and she did not need to nap during the day. Her family history was unremarkable with regard to sleep or circadian rhythm disorders.

Physical examination was unremarkable with the exception of tenderness over the cervical spine and a reduced range of motion of the head owing to neck pain. The level of thyroid stimulating hormone was normal. Nocturnal polysomnography revealed a sleep latency of 12 minutes, sleep efficiency of $88 \%, 18 \%$ slow wave sleep, 13\% REM sleep, and three NREM/ REM sleep cycles. There were no periodic limb movements, and no sleep related breathing disturbances. An actimetric recording was obtained over the course of 19 days and nights. To our surprise, it revealed a short period, free running restactivity cycle (fig 1A). Actigraphy was therefore resumed four days later, before she departed for a group vacation. For 10 days, the group went sightseeing during daytime and rested at night. Thus the patient had a forced regular activity during daytime and the opportunity to sleep at night. At home, she found her daytime sleepiness was significantly improved. Her sleep period was stable thereafter, but later than before the accident (between 1 am and $9 \mathrm{am}$ ). A validated marker of circadian phase, the evening rise in salivary melatonin concentration, occurred at 8:30 $\mathrm{pm}^{.5}$ (fig lB)

As frequent headaches persisted, magnetic resonance (MR) imaging of the brain was carried out 14 months after the accident (fig IC). This revealed a bilobular aneurysm at the bifurcation of the right internal carotid artery, adjacent to the hypothalamic suprachiasmatic nuclei, the seat of the circadian pacemaker ${ }^{6}$ (fig ID). A multiple sleep latency test at that time revealed a mean sleep latency of 19.5 minutes, and no REM sleep occurred. Because of the risk of spontaneous rupture, the aneurysm was occluded by catheter guided embolisation. The patient subsequently still felt some excessive sleepiness but to a much lesser extent than during the initial weeks after the accident. Actigraphy carried out 3.5 years later showed that she continued to have a 24 hour restactivity pattern. She had resumed part time professional activity with a regular daytime schedule. Analysis of clock gene Per3 polymorphism in a blood sample showed that she was homozygous for the four-repeat allele. ${ }^{7}$

\section{DISCUSSION}

We describe a woman with a short period free running circadian rest-activity cycle occurring after a car accident with a whiplash injury. This improved on subsequent resynchronisation induced by a social zeitgeber during two weeks of group travel abroad with regular exposure to daylight during wakefulness and rest periods at night. Clinical examination precluded any other sleep disturbance such as narcolepsy, sleep apnoea, periodic limb movement disorder, or restless legs. Several hypotheses are consistent with the observed sequence of events-for example, occasional cases of post-traumatic delayed sleep phase syndrome have been reported. ${ }^{4}$ The fact that no structural brain lesions were demonstrated by magnetic resonance imaging does not exclude a similar mechanism in our patient. The anatomical vicinity of the internal carotid artery aneurysm to the suprachiasmatic nucleus further raises the possibility that the former affected the functioning of the internal circadian clock. The aneurysm might have occurred during the accident, or subsequently during stress related surges in 


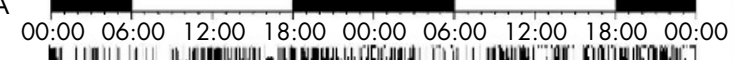
Mo.Di
Di.Mi

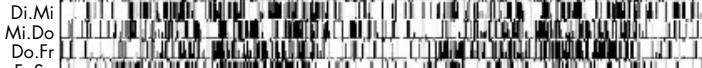

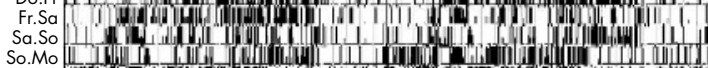

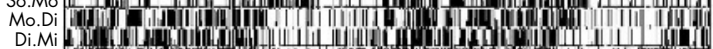

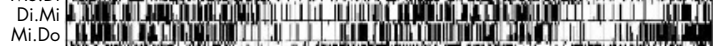

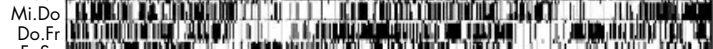
Fr.Sa Sa.So
So.Mo

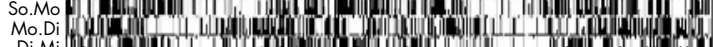

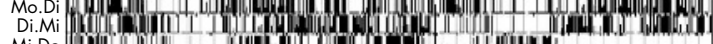

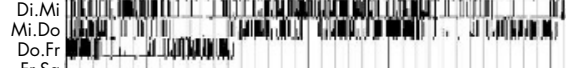

Fr.Sa Sa.So
So.Mo

Mo.Mo

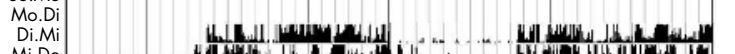

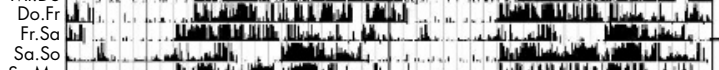

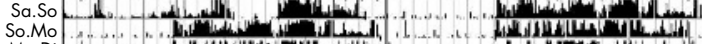

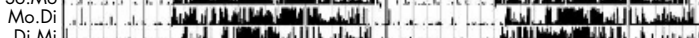

Mi.Do

Do.Fr

Fr.Sa
Sa.So
So.Mo

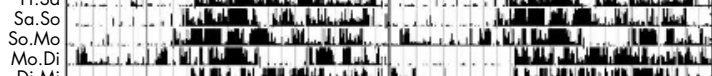

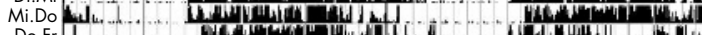

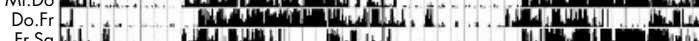

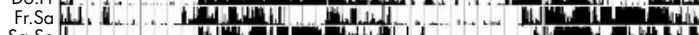

So.Mo Di lin

Di.Mi $\mathrm{Mi}$

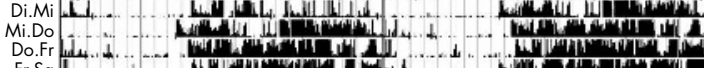

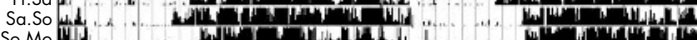

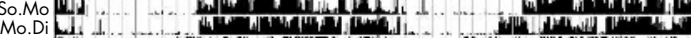

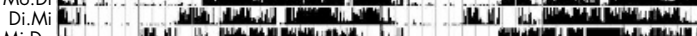

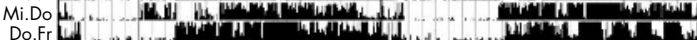

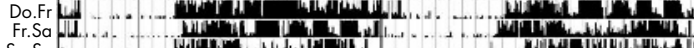

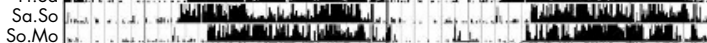

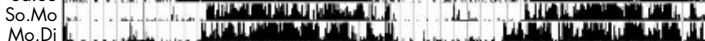

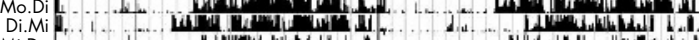

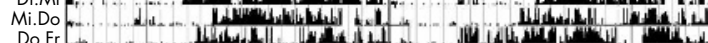

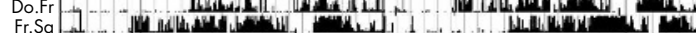

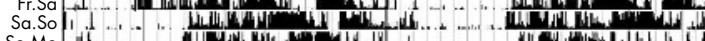

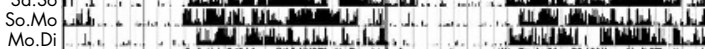

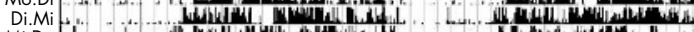

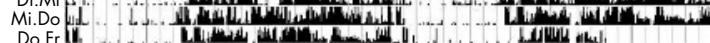
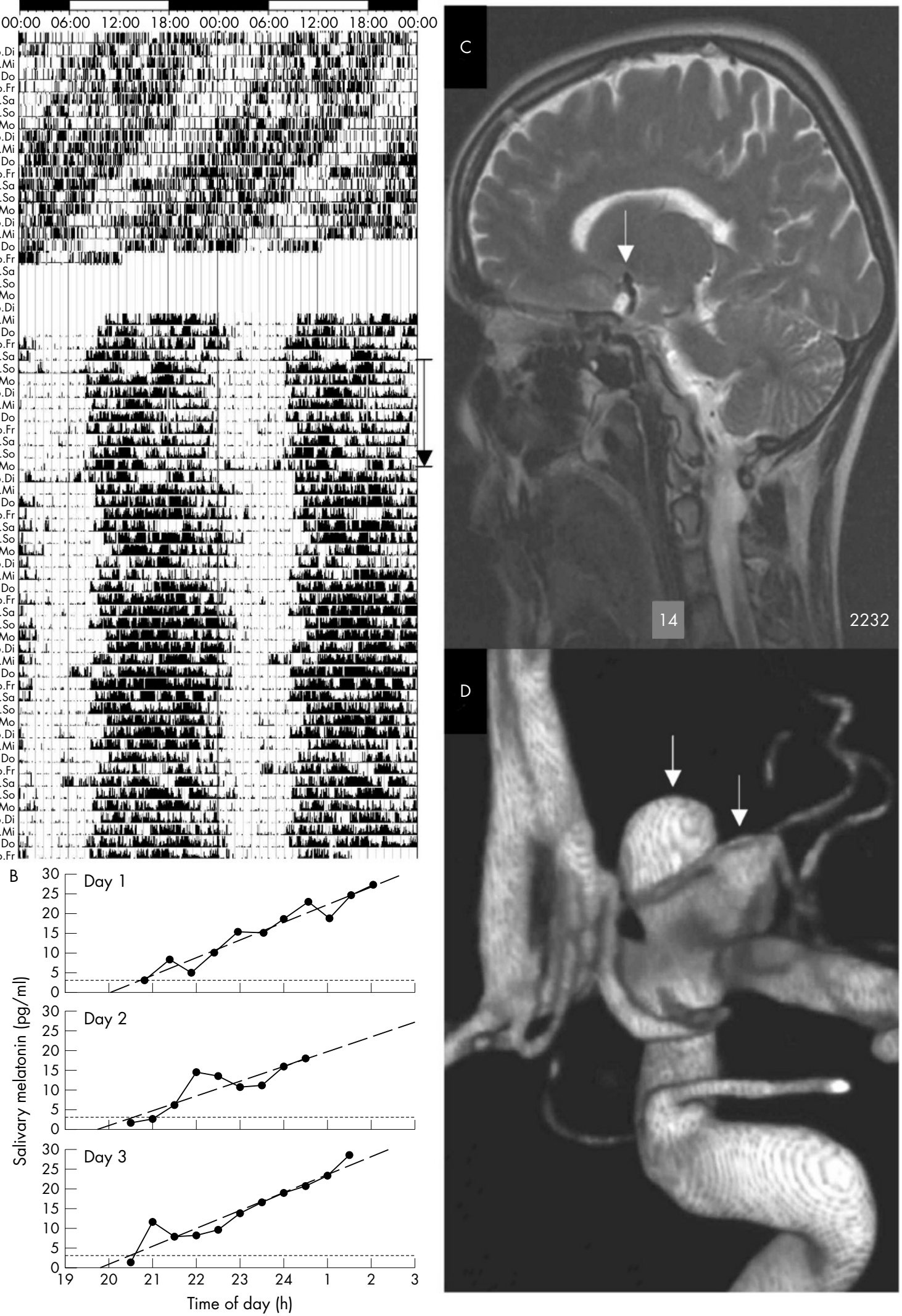

. 
blood pressure, or might have pre-existed. The role of the aneurysm-and its occlusion by catheter embolisation-on the circadian rhythm disturbances therefore remains uncertain. A predisposition to a short circadian period could also be genetic, not manifested before the accident because of her regular job, but emerging during the at-home phase of convalescence. The limited genetic analysis focusing on Per3 polymorphisms did not reveal the five-repeat allele associated with morning preference, but she was four-repeat homozygous which is not specific for any particular diurnal preference. $^{7}$ A potential genetic basis of the clinical manifestations in this patient therefore remains elusive. Improvement of symptoms after the vacation would be consistent with all of the cited potential causes of a transient circadian rhythm disturbance. Under these entrained conditions that followed a period of free run shorter than 24 hours, melatonin onset was slightly early and sleep timing late, resulting in a longer phase angle than usual. This contrasts with the significantly shorter phase angle between melatonin and sleep propensity in patients with a free running rhythm longer than 24 hours. $^{8}$

Our observations suggest that trauma in the region of the suprachiasmatic nuclei induced an unusually short period free running rhythm, which was unmasked by lack of social zeitgebers during convalescence but could be re-entrained by adequate social input. A whiplash injury may result in treatable circadian rhythm disturbances in addition to the more common symptoms of head and neck pain, fatigue, and lack of concentration. Actimetry may be a useful tool in accident patients suffering from daytime sleepiness and nocturnal disturbances, to document the timing and consolidation of sleep objectively.

\section{ACKNOWLEDGEMENTS}

We thank the following for their contributions: R A Cotti, Bühlmann Laboratories, Basle, Switzerland, for performing the melatonin essays; Dr S Archer, Centre for Chronobiology, University of Surrey, UK, for completing the Per3 analysis; Prof Dr G Valavanis, Institute of Neuroradiology, University Hospital of Zurich, Switzerland, for providing the MRI illustrations.

\section{Authors' affiliations}

K E Bloch, T Brack, Sleep Disorders Centre, Pulmonary Division, University Hospital of Zürich, Zürich, Switzerland

A Wirz-Justice, Centre for Chronobiology, Psychiatric University Clinic, Basel, Switzerland

Competing interests: none declared

Correspondence to: Dr Konrad E Bloch, Sleep Disorders Centre, Pulmonary Division, University Hospital of Zürich, Rämistrasse 100, CH8091 Zürich, Switzerland; pneubloc@usz.unizh.ch

Received 21 November 2004

In revised form 21 November 2004

Accepted 23 December 2004

\section{REFERENCES}

1 Schrader H, Bovim G, Sand T. The prevalence of delayed and advanced sleep phase syndromes. J Sleep Res 1993;2:51-5.

2 Dagan Y. Circadian rhythm sleep disorders (CRSD). Sleep Med Rev 2002;6:45-54.

3 Toh KL, Jones CR, He Y, et al. An hPer2 phosphorylation site mutation in familial advanced sleep phase syndrome. Science 2001;291:1040-3.

4 Quinto C, Gellido C, Chokroverty S, et al. Posttraumatic delayed sleep phase syndrome. Neurology 2000;54:250-2.

5 Voultsios A, Kennaway DJ, Dawson D. Salivary melatonin as a circadian phase marker: validation and comparison to plasma melatonin. J Biol Rhythms 1997; 12:457-66.

6 Herzog ED, Schwartz WJ. A neural clockwork for encoding circadian time. J Appl Physiol 2002;92:401-8.

7 Archer SN, Robilliard DL, Skene DJ, et al. A length polymorphism in the circadian clock gene Per3 is linked to delayed sleep phase syndrome and extreme diurnal preference. Sleep 2003;26:413-15.

8 Uchiyama M, Shibui K, Hayakawa T, et al. Larger phase angle between sleep propensity and melatonin rhythms in sighted humans with non-24-hour sleepwake syndrome. Sleep 2002;25:83-8. 